\title{
Current Insights into the Management of Late Chemotherapy Toxicities in Pediatric Osteosarcoma Patients
}

\author{
Stefanie Hecker-Nolting' \\ Thorsten Langer ${ }^{2}$ \\ Claudia Blattmann' \\ Leo Kager ${ }^{3}$ \\ Stefan S Bielack ${ }^{1,4}$ \\ 'Pädiatrie 5 (Onkologie, Hämatologie, \\ Immunologie), Zentrum für Kinder-, Jugend- \\ und Frauenmedizin, Stuttgart Cancer \\ Center, Klinikum Stuttgart - Olgahospital, \\ Stuttgart, Germany; ${ }^{2}$ Pädiatrische \\ Onkologie und Hämatologie, Klinik für \\ Kinder- und Jugendmedizin, \\ Universitätsklinikum Schleswig-Holstein, \\ Campus Lübeck, Lübeck, Germany; \\ ${ }^{3}$ St. Anna Kinderspital, Abteilung für Kinder- \\ und Jugendheilkunde, Medizinische \\ Universität Wien, Vienna, Austria; \\ ${ }^{4}$ Abteilung für Pädiatrische Hämatologie und \\ Onkologie, Klinik für Kinder- und \\ Jugendmedizin - Pädiatrische Hämatologie \\ und Onkologie, Münster, Germany
}

\begin{abstract}
With ever increasing long-term, disease free survival rates, long-term toxicities of otherwise successful therapy have gained increasing importance. They can be grouped into potentially life-threatening, especially secondary malignancies and anthracycline cardiomyopathies, potentially disabling, particularly severe hearing loss and renal insufficiency, other, and rare events. Pathophysiology, frequency, and medical treatment approaches are discussed. Finally, fertility issues and quality of life issues are discussed, together with an outlook into the future. The challenge to cure as many patients as possible from osteosarcoma while enabling a life free of late effects will remain.
\end{abstract}

Keywords: osteosarcoma, child, adolescent, chemotherapy, late effects

\section{Introduction}

Osteosarcoma, the most frequent bone cancer of the young, carries an extremely high risk of metastases, which are usually pulmonary. The formerly almost always fatal disease course was only changed with the introduction of first adjuvant, then neoadjuvant chemotherapy into treatment in the late 1970s and early 1980s. Nowadays, some $70 \%$ percent of affected children and adolescents are made long-term, disease free survivors by a combination of local, mostly surgical, and systemic therapies. ${ }^{1}$

Over the years, a plethora of studies has been performed to find the optimal antineoplastic medications. From these, doxorubicin, high-dose methotrexate, cisplatin, and, to a lesser extent, ifosfamide have emerged as the most active. Others such as cyclophosphamide, carboplatin, etoposide, and still others are used much less frequently and rarely first-line. ${ }^{1}$ More recently, other, targeted drugs have also shown some efficacy. ${ }^{2,3}$ So far, this has been confined to the relapse setting and to very limited prolongations of survival, while integration into first-line therapies still drags behind. Accordingly, most long-term survivors will have been treated with the more conventional drugs and surgery. This review tries to cover the most common late side effects of medical osteosarcoma therapy and avenues towards their effective treatment. \section{Pädiatrie 5 (Onkologie, Hämatologie,}

Correspondence: Stefan S Bielack Immunologie) Zentrum für Kinder-, Jugend- und Frauenmedizin, Stuttgart Cancer Center, Klinikum Stuttgart Olgahospital, Kriegsbergstr. 62, Stuttgart, 70174, Germany

Tel +497 II 2787388 I

Fax+49711 2787-3882

Email coss@klinikum-stuttgart.de

\section{Life-Threatening Late Effects Secondary Malignancies}

The diagnosis of osteosarcoma is already a major blow to the patient and his or her family. A second, even more devastating blow may arise from the diagnosis of 
a second malignancy. This may be a leukemia, where the additional risk is largely confined to the first ten years after osteosarcoma, or a secondary solid cancer, where no such time limitation is observed. ${ }^{4}$ Taken together, second malignancies have been reported as being the most frequent cause of late death after the original malignancy. ${ }^{5}$ In one large competing risk meta-analysis of 72,945 connective tissue malignancy patients with a median follow-up of 131 months, osteosarcoma histology was associated with an increased risk of secondary acute leukemia at a hazard ratio of $5.06 .{ }^{6}$ Chemotherapy, exposure to radiotherapy or even diagnostic irradiation, ${ }^{7}$ and individual cancer predisposition may all contribute to the development of secondary malignancies. In addition to medical therapy of the original malignancy, factors predisposing to cancer may be present and causative for this severe complication. ${ }^{8}$

Secondary leukemias are much more likely to be of the myeloid than of the lymphoblastic subtype. They tend to be acute rather than chronic and can broadly be classified into two types, alkylator related and topoisomerase IIinhibitor related leukemias ${ }^{9,10}$ (Table 1). Since their advent well over half a century ago, akylators - here usually ifosfamide - have been a classical risk factor for the development of secondary leukemias. Their median time from osteosarcoma is around 5-7 years. Alkylator-related leukemias are commonly acute and non-lymphocytic. They often arise from a pre-leukemic, myelodysplastic phase and carry numerical chromosomal abnormalities.
Among these, monosomy 7, trisomy 8 , and $5 \mathrm{q}$ - are the most prominent. ${ }^{11}$ Topoisomerase II associated leukemias, on the other hand, develop earlier, only a few years from primary therapy. ${ }^{12}$ Among the drugs more commonly employed against osteosarcoma, the epipodophyllotoxin etoposide (VP16), but also the anthracycline doxorubicin, which seems to exert at least some of its activity via topoisomerase II $^{13}$ have been held responsible. These secondary leukemias are also myeloid, but generally of the monoblastic or myelomonoblastic variant and have no myelodysplastic prephase. Other than their alkylator associated counterparts, they carry translocations rather than numerical chromosomal changes. Translocations involving the lysine methyltransferase $2 \mathrm{~A}$ (KMT2A, formerly mixed linea leukemia $M L L$ ) gene on chromosome region $11 \mathrm{q} 23$ are by far the most frequent, ${ }^{11}$ but others may also occur.

Unfortunately, the prognosis for both types of secondary leukemia remains extremely poor, even with intensive therapies such as allogeneic bone marrow transplantation. ${ }^{14}$ Avoidance of the drugs most likely responsible for this potentially fatal complication seems the only feasible protective measure. This may be achieved in case of etoposide, for which potent alternatives are present, or alkylators, which may be also be substituted by other drugs. It is not well feasible for the anthracycline doxorubicin, for which most experts see no reasonable alternative. After all, the osteosarcoma has to

Table I The Two Major Types of Chemotherapy Induced Secondary Leukemia

\begin{tabular}{|c|c|c|}
\hline Leukemia Type & Acute Myeloid & Acute Myelomonoblastic or Monoblastic \\
\hline Median latency & $5-7$ years & $\mathrm{I}-3$ years \\
\hline Main predisposing agents & $\begin{array}{l}\text { Alkylators } \\
\text { - ifosfamide } \\
\text { - cyclophosphamide }\end{array}$ & $\begin{array}{l}\text { Topoisomerase II inhibitors } \\
\text { - etoposide } \\
\text { - anthracyclines (?) }\end{array}$ \\
\hline Prephase & Myelodysplastic & None \\
\hline Chromosomal alterations & $\begin{array}{l}\text { Numerical alterations } \\
\text { - monosomy } 7 \\
\text { - trisomy } 8 \\
\text { - 5q- } \\
\text { - others }\end{array}$ & $\begin{array}{l}\text { Translocations } \\
\text { - mostly involving I Iq23 (MLL-gene) } \\
\text { - others }\end{array}$ \\
\hline Prognosis without treatment & Fatal & Rapidly fatal \\
\hline Treatment with curative intent & Bone marrow transplant & Bone marrow transplant \\
\hline Prognosis with such treatment & Very poor & Very poor \\
\hline
\end{tabular}


be cured in order to contract any late complications in the first place. Therefore, causative agents cannot be simply removed from osteosarcoma therapy without reasonable substitutes.

Secondary solid cancers may also occur after osteosarcoma. Their risk appears to be life-long. In fact, osteosarcoma itself ranks among the most common secondary malignancies after childhood cancer. ${ }^{15,16}$ It should also be noted that presumed osteosarcoma metastases to sites like distant bones may indeed represent a secondary cancer. ${ }^{17}$ Radiotherapy, the most prominent risk factor for secondary solid cancers in many other secondary cancers, will have been only rarely administered to osteosarcoma survivors. Hence, it is much more likely that anticancer drugs or a cancer predisposition syndrome were responsible.

The true frequency of cancer predisposition syndromes in osteosarcoma remains a matter of debate, but it is likely to be considerable. ${ }^{18}$ It has thus been argued that all osteosarcoma patients be screened for such genetic cancer predispositions.

If it arises, a secondary solid cancer merits therapy. Therapeutic nihilism is contraindicated. Prognosis will greatly depend on its type, site, and stage and cannot be generalized. Avoidance of those drugs most commonly responsible for secondary malignancies from osteosarcoma therapy seems to offer very effective protection. If, as more often than not, not feasible, these agents should be administered at as low a cumulative dose as possible. Unfortunately, there are no other known protective measures. Close observation to detect these potentially fatal, but also potentially treatable and curable second cancers at an early stage seems wise.

\section{Heart Failure}

Doxorubicin remains one of the most important, if not the most important of all drugs against osteosarcoma. ${ }^{19}$ Its administration, however, does not come without its price: Anthracycline induced heart failure is one of the most devastating late effects of otherwise successful chemotherapy. It comes in the form of dilated cardiomyopathy. This side-effect is not limited to the time immediately after treatment, quite to the contrary: Heart function seems to decrease with the years and overt heart failure may occur even decades after therapy. ${ }^{20}$ As many childhood cancer survivors will have been exposed to anthracyclines, the risk is substantial. It has been estimated that over 50.000 cases of doxorubicin cardiomyopathy would be present globally in young cancer survivors in $2020 .^{21}$ Unfortunately, the assumption is that less than half of those who develop such chemotherapy-related toxicity will survive beyond 10 years. ${ }^{22}$ Ifosfamide, especially in high dosages, may also rarely result in heart failure, but this seems to be an acute problem, very rare, and will not be discussed further on this occasion.

Given the magnitude and severity of this devastating late effect, the number of published articles dealing with this severe complication is almost uncountable, with almost 5.000 papers on "doxorubicin cardiotoxicity" in medline alone. Still, the pathophysiology of anthracyclineinduced cardiomyocyte damage remains the subject of scientific (and sometimes heated) discussions. Comprehensive reviews are available in the recent literature. As a consensus, it seems that the formation of free radicals exceeding the hearts detoxification capacity plays a major role, but other mechanisms may be active, at least in addition. ${ }^{21,23-25}$

With anthracycline cardiomyopathy being such a devastating late effect, potential risk factors have long been of major interest. For one, traditional cardiovascular risk factors such as hypertension, diabetes, dyslipidemia, and overweight or obesity should not be disregarded. ${ }^{26}$ Very young and very old patients may be at an enhanced risk, as may be females over males. Variations of a child's genetic make-up may also contribute to his or her risk for later cardiomyopathies. ${ }^{27}$ The major predisposing factor for late chemotherapyrelated cardiomyopathy, however, seems to be the cumulative anthracycline dose administered. Regimens are therefore usually limited at $450 \mathrm{mg} / \mathrm{m}^{2}$ doxorubicinequivalent, where congestive heart-failure is in the range of $5 \%$. Lower cumulative doses seem to carry lower risks, but even the lowest doses may not be totally cardioprotective. ${ }^{28}$

It has been demonstrated decades ago that the risk for anthracycline cardiotoxicity was also drug schedule dependent, large single doses being more cardiotoxic than the same drug dose being split up over several days or weeks of administration. Continuous infusions also seem to be less cardiotoxic than bolus scheduling. ${ }^{29}$ The latter, however, has been disputed for the youngest patients, one of the most vulnerable populations. In these, a randomized study of $360 \mathrm{mg}$ cumulative doxorubicin found no late cardiac benefits for infusions over 48 hours compared to bolus administration. ${ }^{30}$ The situation may differ in 
adolescents, young adults, and older patients. However, randomized evidence for these age groups is lacking.

Females may be at a higher risk for late cardiac damage than males. This is particularly relevant when young women are about to plan building a family: Pregnancy and childbirth pose additional stresses to the heart. The demands on cardiac output are increased substantially. Cardiac decompensation may occur even in the face of previously undetectable damage. ${ }^{31}$ It seems wise to counsel accordingly, treat if necessary, and to follow pregnancies extremely closely.

Given the severity of this problem, prevention has become a major point of interest and of study. So far, there are no methods for complete cardiomyopathy avoidance other than complete anthracycline abstinence - often not feasible for oncological reasons. The current knowledge about anthracyline treated patients was comprehensively reviewed a few years ago. ${ }^{26}$ As a rule, primary prevention may be the most effective strategy, with multiple options being explored. ${ }^{32}$ Alternative drugs with lower cardiotoxic potential, particularly anthracycline analogues or anthrachinones, have yet to prove that they are as effective against osteosarcoma as doxorubicin. It has been well documented that anthracycline peak levels correlate with untoward effects upon the heart, at least in adolescent and adults. $^{29}$ Hence, drug scheduling has received quite some attention. Both split dosages and continuous infusions of up to 96 hours or even longer were shown to protect the heart. ${ }^{28}$ However, these modified schedules may be significantly more toxic to the mucous membranes than is bolus administration, so that this does not come without its acute price. As mentioned previously, anthracycline scheduling has been studied in detail in pediatric leukemia, the patient population being considerably younger than that for osteosarcoma. In these young children, 48 hour infusions demonstrated no cardiac benefits over bolus administration. ${ }^{30}$

Over the years, much interest has been focused on the cardioprotectant dexrazoxane. ${ }^{33}$ The agent is believed to diminish the heart of free radicals, favored by anthracycline therapy, and associated oxidative stress and cardiac destruction. Where it is licensed, its indication generally only starts at a certain cumulative anthracycline dose, is limited to adults, and to certain cancer types other than osteosarcoma. Osteosarcoma patients may only be treated off-label. Recently, the Children's Oncology Group reported on favorable long-term cardiac outcome data with dexrazoxane in pediatric osteosarcoma patients receiving large cumulative amounts of doxorubicin (375$\left.600 \mathrm{mg} / \mathrm{m}^{2}\right) .{ }^{34}$ In 2017, the European Medicines Agency agreed that the existing pediatric contraindication for cardioxane be lifted for patients under 18 years who require high doses of anthracyclines (ie, $>300 \mathrm{mg} / \mathrm{m}^{2}$ ) and are therefore considered at greater risk of harmful cardiac effects. Therefore, dexrazoxane may be beneficial for children and adolescents, so that further study in these young patients is warranted. Other drugs used for primary cardiac prevention include statins, but randomized evidence for this specific situation is lacking. The same holds true for non-medicinal primary prevention such as exercise. ${ }^{26}$ Further studies are clearly required.

Secondary prevention of anthracycline-induced cardiotoxicity has also gathered quite some scientific interest. Beta-blockers, aldosterone antagonists, angiotensin receptor blockers, angiotensin converting enzyme inhibitors, neurohormonal blocking drugs and implantable devices have and may still all be used. However, most publications deal with heart-failure of other or of all causes and evidence-based experience for this specific situation is scarce to non-existent. ${ }^{26}$

It has been argued that cardiac therapy should be initiated immediately once subclinical signs of heart failure became evident by echocardiography or other testing methods. The optimal therapy, however, is anything but well defined. A Cochrane review 2016 found only two evaluable trials, one of phosphocreatine and one of the acetyl choline esterase inhibitor, enalapril. Unfortunately, neither drug managed to prove any long-lasting benefit. ${ }^{35}$ Still, many cardiologists will treat patients with asymptomatic (or even symptomatic) terminal heart failure just as those with cardiac failure from other causes. Refractoriness to even the most active drugs is, however, all too frequent.

In such refractory cases, allogeneic hearttransplantation may become necessary, sometimes with external bridging devices. It is of some consolation that the prognosis of heart-transplants in long-term cancer survivors need not be inferior to that of other patients. ${ }^{36,37}$ There seems to be no increased risk for malignant recurrences with these transplants, so that such an invasive therapy may be very well indicated.

It has been repeatedly recommended that all or at least most anthracycline treated pediatric patients be screened and, if indicated, treated for cardiomyopathy, but many associated factors are still the focus of intense discussion. Consensus recommendations, however, are available. ${ }^{26,38}$ 
An international expert panel managed to come up with the following unanimous opinions: ${ }^{26}$

- Childhood cancer survivors treated with anthracyclines (including mitoxantrone) or chest radiation are at increased risk of cardiomyopathy.

- Surveillance using echocardiography should be lifelong and performed at a minimum of every five years.

- Given the increased cardiometabolic demand on the heart of the mother during pregnancy, closer monitoring of survivors during pregnancy is warranted.

- Survivors with documented asymptomatic cardiomyopathy should be referred to a cardiologist for further diagnostic work-up and possible treatment.

- At risk cancer survivors should be regularly screened for traditional cardiovascular risk factors (ie, hypertension, diabetes, dyslipidemia, overweight/obesity) and should be counseled against smoking and physical inactivity.

\section{Disabling Late Toxicities Mobility}

Many former osteosarcoma patients are considerably handicapped by surgery for their primary tumor, which more often than not resulted in loss of at least one major joint. Chemotherapy, however, seems to add little to this loss of mobility other than that it may hinder the initiation of rehabilitative measures. Endoprostheses may be the subject of deep infection. Once chemotherapy is over, however, the infection rate drops and the prosthesis is more endangered by mechanical wear and failure. ${ }^{39,40}$

\section{Hearing Loss}

Cisplatin (and to a much lower extent carboplatin) is a major cause of high-frequency hearing loss. It is caused by largely irreversible damage to the hair cells of the inner ear. Typically, this begins at the highest frequencies and then progresses to the lower frequencies. In the end, it may cause permanent difficulties to understand the spoken word and, rarely, near-complete deafness. ${ }^{41,42}$

Hearing loss is greatly dependent, among other factors, on the cumulative cisplatin dose administered. It may therefore increase as treatment progresses. Other ototoxic drugs such as aminoglycosides and also noise may aggravate the situation, particularly if present concomitantly with cisplatin. Younger age at cisplatin exposure has also been implied as a risk-factor. ${ }^{43,44}$
Approaches to limit this inner ear damage include trying to reduce exposure to high peak cisplatin concentrations by intelligent drug scheduling, be it prolonged infusions or administration as split dosages. Less ototoxic platinum analogues have yet to prove their equi-efficacy to cisplatin. Otoprotective drugs such as amifostine, sodium diethyldithiocarbamate disulfiram, or systemic sodium thiosulfate have been found more or less active in some other cancers. ${ }^{45}$ However, these drugs have not been well evaluated in the osteosarcoma population. Some may even be contraindicated. Amifostine, for example, is a prodrug activated by alkaline phosphatase, an enzyme often amply present in osteosarcoma. The drug may therefore protect not only the inner ear but also the tumor itself from cisplatin's effect.

Once severe ototoxicity has arisen, it is important to help with understanding of the spoken word, as this will greatly support a child's development. The indication for hearing aids should therefore be applied liberally. ${ }^{41}$ Drugs will not be able to restore any damage once it has occurred, it will remain permanent.

\section{Kidney Function}

The kidney may be the focus of cisplatin or, rarer, ifosfamide-associated glomerular dysfunction and of ifosfamide-related tubular toxicity. ${ }^{46}$ Methotrexate associated glomerular toxicity may be extremely severe. It can lead to renal failure and concomitant loss of drug excretion, which may lead to toxic death. However, methotrexate induced renal damage is always acute and renal function will return to completely normal once the acute episode is survived. $^{47}$ After osteosarcoma, dialysis and renal transplantation are rarities, as permanent glomerular failure is extremely infrequent. If they occur, other causes must be sought. A causative impact of former chemotherapy should only assumed if none are detectable The function of the renal glomerulus may, however, be less severely impeded by anti-tumor chemotherapy, the kidney being well able to tolerate this.

Clinically relevant renal tubular toxicity occurs much more frequently than glomerular failure. It is usually associated with ifosfamide use and may be permanent. ${ }^{46}$ Ifosfamde's cumulative dose and a preexisting reduced number of renal glomeruli (hence reduction of the glomerular filtration rate GFR, eg, following unilateral nephrectomy) are well-defined risk factors for enhanced ifosfamide-related damage. As younger children have a comparatively lower GFR, they are at an increased 
risk. Previous or concomitant cisplatin therapy works through the same GFR-effect.

Tubular toxicity is characterized by a loss of small substrates through the urine rather than uremia. In extreme cases, a full-blown De Toni-Debré-Fanconi's syndrome may result. Glucosuria, phosphaturia, aminoaciduria, and type II renal tubular acidosis may result, with associated hypokalemia, sodium wasting, and dehydration. ${ }^{48}$ It is therefore pertinent that osteosarcoma patients whose therapy included ifosfamide be screened for such urinary losses, usually for several years after therapy. Measuring the kidney's fractional absorption rate of phosphate seems to offer a good screening tool. ${ }^{49}$ Those detected to show abnormalities merit a more detailed evaluation by a nephrologist. Patients treated without ifosfamide generally have little to no demand for such renal screening. Therapy mirrors that of renal tubular damage in general and involves oral substitution of substances lost through the urine. In particular, phosphate and bicarbonate may have to be permanently substituted in order to prevent consecutive problems. N-acetylcysteine may have some, as of yet poorly defined role as a renal protectant from ifosfamide, ${ }^{50}$ but is not in widespread use for this purpose.

\section{Rare Late Effects}

\section{Brain}

Osteosarcoma chemotherapy is usually not associated with clinically apparent late toxicities to the brain. Methotrexate encephalopathy may occur acutely, but is generally reversible. Investigators have, however, claimed some lasting intelligence limitations associated with the drug's use in children, ${ }^{51}$ particularly if administered at high dosages. There is little knowledge gathered about osteosarcoma patients in particular, but these may be at an enhanced risk because of the high individual and cumulative methotrexate doses received. Acute methotrexate damage to the brain may be a harbinger for late damage. However, studies have generally been performed in survivors of acute leukemias and little is known about osteosarcoma, as is about specific therapy.

\section{Peripheral Nerves}

The peripheral nerves may be the focus of severe, acute damage caused by cisplatin. ${ }^{52}$ This only very rarely affects the younger patients who most frequently contract osteosarcoma, it is the aged who are most susceptible. The rare older or even geriatric osteosarcoma patient may, however, experience peripheral neurotoxicity to an extent at which it can be dose-limiting. Here, time is a healer rather than an aggravator, so that late neurotoxicity is so far not of major concern.

\section{Lungs}

The lungs are another organ system which seems to be quite safe from late chemotherapy toxicities. Reductions of pulmonary function, if present, are much more likely to be caused by former surgery or, rarely, radiotherapy for lung metastases than by chemotherapy.

\section{Liver}

While many organs are affected by late toxicities, the liver seems to be relatively safe. In the absence of transfusionrelated infectious hepatitis, there is no need for concern. Late hepatic problems are more likely to have some other cause than osteosarcoma chemotherapy, for instance transfusion-related hepatitis.

\section{Teeth}

Dental abnormalities may occur after treatment for childhood cancer, they are not osteosarcoma specific. Most late effects are related to tooth development during dentition. ${ }^{53}$ Judging from osteosarcoma's age distribution, most affected patients will have developed permanent teeth by the time chemotherapy is given, thereby sparing this organ from most relevant late effects.

\section{Immune System and Vaccinations}

The body's immune system and its associated protection against infections generally both return to completely normal within several months to one year after chemotherapy. Of importance, however, protection by vaccination may be impeded during and after intensive chemotherapy. It therefore seems advisable to test antibody titers for those diseases against which a patient was formerly vaccinated (for instance measles, mumps, rubella, varicella, diphtheria, tetanus, pneumococcus, hepatitis A \& B, and sometimes others) several months after the end of chemotherapy and to re-vaccinate in case of negativity. ${ }^{54-57}$ Hepatitis vaccination is always indicated in the absence of appropriate titers, as chemotherapy and concomitant transfusions may again become indicated in case of osteosarcoma relapse. Varicella vaccination may prevent the otherwise frequent virus reactivation as shingles. If shingles do develop, however, they need to be treated, usually by acyclovir. ${ }^{58}$ There is no hint of long-lasting or even permanent, clinically significant immune damage after osteosarcoma chemotherapy. 


\section{Other Rare Late Toxicities}

Other tissues and organs may only infrequently be affected by late effects or may even be completely spared from such. For example, major hair loss is rarely if ever a permanent problem. The same holds true for skin alterations, with the exemption of surgical scars. The mucosa, while a major focus of acute chemotherapy toxicities, usually recovers fully within weeks. The nose and sense of smell, which may be affected by acute mucositis, return to normal with recovery, as does taste. Vision is also more or less unaffected by current osteosarcoma therapies. Strength returns to normal with increased physical activity, as does bone mass. There are no known major late effects affecting the mucosa of the digestive tract. The list could go on and on. Treatment is of little relevance and of little interest. If presumed late toxicities not mentioned in this review are suspected, the pertinent literature should be sought.

\section{Fertility}

Former osteosarcoma patients are most often in or coming into their reproductive years. They may be concerned about fertility and ask about potential tumor predisposition in their potential offspring. Many concerns may be rightly dissolved, others are indeed relevant.

Recommendations concerning fertility preservation in cancer patients are available. ${ }^{59}$ Fertility is more often than not quite normal in females, who may enjoy what seems to be a nearly normal reproductive rate. Males, however, may experience major treatment-related impairments. This holds particularly true for those males who received high cumulative doses of alkylators (ifosfamide, cyclophosphamide) during osteosarcoma treatment. Those few osteosarcoma patients whose gonads were exposed to radiotherapy with even a few Gy will also experience greatly reduced fertility, males again more severely affected than females. Therapy in case of impeded male fertility mirrors infertility treatments in others. Assisted reproduction techniques may become necessary in selected young survivors, adoption may be an option for some.

Females should be advised that, while their menstrual cycle seems to have returned to normal only a few months after the end of treatment, their reproductive age could end prematurely. In one study of 1067 women surviving any type of cancer for 5 years or more, in whom cancer was diagnosed before age 20, and who were still menstruating at age 21 , increased relative risks of menopause were detected after treatment with either radiotherapy (relative risk 3.7) or alkylating agents (relative risk 9.2). The risk of menopause increased 27-fold for women treated with both radiotherapy below the diaphragm and alkylating agents. By age $31,42 \%$ of these women were infertile. ${ }^{60}$ Treatment may be less gonadotoxic by now and osteosarcoma therapy is devoid of the most gonadotoxic agents anyhow. Still, it may be advisable for young, fertile women not to postpone planned pregnancies for all too long. Then, assisted reproduction technique or egg banking will generally not be necessary. Otherwise, oocyte cryopreservation may offer a viable alternative.

One concern can be rightly taken from most former patients, be they male or female: Their offspring will not have an increased risk of treatment-induced malformations, it will be quite normal (caveat: rare tumor predisposition syndromes may also be associated with malformations). Also, former chemotherapy will not cause cancer in the offspring. Children may, of course, have inherited their parents' tumor predisposition syndromes, if present. Once children have been born, they are not at risk of any other untoward late effects of their parents' former chemotherapy, whatever those might have been. One caveat is in utero exposure, which would require medical attention to the child.

\section{Quality of Life}

With all these early and late chemotherapy toxicities - in association with a more or less disabling tumor surgery - it comes as no surprise that the quality of life and patientreported outcomes of osteosarcoma patients have recently come under increased scrutiny. ${ }^{61-64}$ It is most often not possible to unravel which specific treatment caused which specific long-term impediment. Resulting changes may be so negative to pose seemingly insurmountable problems or may even be experienced as positive. On the negative side, survivors may for example be affected by the metabolic syndrome. It will remain virtually impossible to unravel whether chemotherapy or surgery or the combination of both was mainly responsible. Never the less, it seems wise to counsel survivors about this specific late effect and about methods of prevention. ${ }^{65,66}$

The results of the international large prospective EURAMOS study, which included a dedicated quality of life assessment alongside and after treatment, are long overdue and more than desperately expected. ${ }^{67}$ They may finally be able to shed a comprehensive light on this subject. 


\section{Outlook into the Future}

Currently, a vast variety of drugs is being evaluated against recurrent osteosarcoma. ${ }^{68,69}$ Few patients among those exposed will have the fortune to become long-term, disease-free survivors and therefore be able to experience late effects. One example of drugs hyped by some is liposomal muramyl-tripeptide phenolamine (MTP), ${ }^{70}$ which is used as part of frontline therapy by a minority of clinicians. The drug, while associated with acute toxicities, seems largely devoid of late effects. Its efficacy, however, remains a major point of controversy. ${ }^{71}$ More recently, multiple targeted therapies have been tested in recurrent osteosarcoma, with some evidence of activity. ${ }^{2,68}$ Tyrosine kinase inhibitors have gained the most attention, as they were shown to prolong disease control in the inoperable relapse situation. Transfer into first-line therapy and into increased cure rates is, however, pending. These or other new agents will undoubtedly lead to new late effects, which will then require investigation and treatments. $^{72}$ A watchful eye is certainly advisable.

\section{Conclusion}

Late toxicities of systemic osteosarcoma therapies can be severe, impede a patient's quality of survival, and may even kill. Like in all predominantly pediatric and adolescent malignancies, emphasis on long-term morbidity and mortality of therapy is important to identify high-risk survivors and to design interventions aimed at early detection and prevention of late toxicities. ${ }^{73}$ They require close, qualified attention. A comprehensive screening recommendation by the Late Effects Surveillance System of the German Society for Pediatric Oncology and Hematology $\mathrm{GPOH},{ }^{74}$ focusing on standard regimens and drugs, is given in Appendix 1. New treatments will undoubtedly also bring new late side effects. Nevertheless, effective systemic therapy is the only chance to beat this cancer. We must all learn to detect, reduce, and treat the associated late effects.

\section{Acknowledgments}

We are indebted to all former and present osteosarcoma patients who helped us learn about late therapy toxicities.

\section{Disclosure}

Stefanie Hecker-Nolting report that her institution received financial support from EISAI. Thorsten Langer, and Claudia Blattmann report no conflicts of interest. Leo Kager reports financial compensation for advisory board roles for Bayer, Novartis, Amgen, and Agios, outside of the scope of this work. Stefan S Bielack reports financial compensation for advisory board roles for Bayer Healthcare, Boehringer Ingelheim, Hoffmann-La Roche, Ipsen, Eli Lilly, and Sensorion, all outside of the scope of this work. The authors report no other conflicts of interest in this work.

\section{References}

1. Bielack S, Cable MG, Gorlick R, et al. Osteosarcoma - approach to therapy. C. A. S. Arndt (ed.), Sarcomas of bone and soft tissues in children and adolescents. Pediatr Oncol. 2021:91-109. doi:10.1007/ 978-3-030-51160-9_8

2. Gaspar N, Marques da Costa ME, Fromigue Oet al. Recent Advances in Understanding Osteosarcoma and Emerging Therapies. Fac Rev. 2020;9:18. doi:10.12703/r/9-18

3. Just MA, Van Mater D, Wagner LM. Receptor tyrosine kinase inhibitors for the treatment of osteosarcoma and Ewing sarcoma. Pediatr Blood Cancer. 2021;24:e29084. doi:10.1002/pbc.29084

4. Hawkins M, Bhatia S, Henderson TO, et al. Subsequent primary neoplasms: risks, risk factors, surveillance, and future research. Pediatr Clin North Am. 2020;67(6):1135-1154. doi:10.1016/j. pcl.2020.07.006

5. Möller TR, Garwicz S, Barlow L, et al. Decreasing late mortality among five-year survivors of cancer in childhood and adolescence: a population-based study in the Nordic countries. J Clin Oncol. 2001;19(13):3173-3181. doi:10.1200/JCO.2001.19.13.3173

6. Sanford NN, Martin AM, Brunner AM, et al. Secondary acute leukemia in sarcoma patients: a population-based study. Int J Radiat Oncol Biol Phys. 2018;100(3):687-694. doi:10.1016/j.ijrobp.2017.11.011

7. Hall EJ, Brenner DJ. Cancer risks from diagnostic radiology. $\mathrm{Br}$ J Radiol. 2008;81(965):362-378. doi:10.1259/bjr/01948454

8. Kratz CP, Jongmans MC, Cavé H, et al. Predisposition to cancer in children and adolescents. Lancet Child Adolesc Health. 2021;5 (2):142-154. doi:10.1016/S2352-4642(20)30275-3

9. Winer ES. Secondary acute myeloid leukemia: a primary challenge of diagnosis and treatment. Hematol Oncol Clin North Am. 2020;34 (2):449-463. doi:10.1016/j.hoc.2019.11.003

10. Oliai C, Schiller G. How to address second and therapy-related acute myelogenous leukaemia. $B r J$ Haematol. 2020;188(1):116-128. doi:10.1111/bjh.16354

11. Higgins A, Shah MV. Genetic and genomic landscape of secondary and therapy-related acute myeloid leukemia. Genes. 2020;11(7):749. doi:10.3390/genes 11070749

12. Bhatia S. Therapy-related myelodysplasia and acute myeloid leukemia. Semin Oncol. 2013;40(6):666-675. doi:10.1053/j. seminoncol.2013.09.013

13. Van der Zanden SY, Qiao X, Neefjes J. New insights into the activities and toxicities of the old anticancer drug doxorubicin. FEBS J. 2020. doi:10.1111/febs. 15583

14. Boddu P, Kantarjian HM, Garcia-Manero G, et al. Treated secondary acute myeloid leukemia: a distinct high-risk subset of AML with adverse prognosis. Blood Adv. 2017;1(17):1312-1323. doi:10.1182/ bloodadvances. 2017008227

15. Schwartz B, Benadjaoud MA, Cléro E, et al. Risk of second bone sarcoma following childhood cancer: role of radiation therapy treatment. Radiat Environ Biophys. 2014;53(2):381-390. doi:10.1007/s00411-013-0510-9

16. Schonfeld SJ, Merino DM, Curtis RE, et al. Risk of second primary bone and soft-tissue sarcomas among young adulthood cancer survivors. JNCI Cancer Spectr. 2019;3(3):pkz043. doi:10.1093/ jncics/pkz043 
17. Franke M, Hardes J, Helmke K, et al. Solitary skeletal osteosarcoma recurrence. Findings from the Cooperative Osteosarcoma Study Group. Pediatr Blood Cancer. 2011;56(5):771-776. doi:10.1002/pbc.22864

18. Mirabello L, Zhu B, Koster R, et al. Frequency of pathogenic germline variants in cancer-susceptibility genes in patients with osteosarcoma. JAMA Oncol. 2020;6(5):724-734. doi:10.1001/ jamaoncol.2020.0197

19. Smith MA, Ungerleider RS, Horowitz ME, et al. Influence of doxorubicin dose intensity on response and outcome for patients with osteogenic sarcoma and Ewing's sarcoma. J Natl Cancer Inst. 1991;83(20):1460-1470. doi:10.1093/jnci/83.20.1460

20. Franco VI, Lipshultz SE. Cardiac complications in childhood cancer survivors treated with anthracyclines. Cardiol Young. 2015;25 (S2):107-116. doi:10.1017/S1047951115000906

21. Mancilla TR, Iskra B, Aune GJ. Doxorubicin-induced cardiomyopathy in children. Compr Physiol. 2019;9(3):905-931. doi:10.1002/ cphy.c180017

22. Towbin JA, Lowe AM, Colan SD, et al. Incidence, causes, and outcomes of dilated cardiomyopathy in children. JAMA. 2006;296 (15):1867-1876. doi:10.1001/jama.296.15.1867

23. Bhagat A, Kleinerman ES. Anthracycline-induced cardiotoxicity: causes, mechanisms, and prevention. Adv Exp Med Biol. 2020;1257:181-192. doi:10.1007/978-3-030-43032-0_15

24. Rawat PS, Jaiswal A, Khurana A, et al. Doxorubicin-induced cardiotoxicity: an update on the molecular mechanism and novel therapeutic strategies for effective management. Biomed Pharmacother. 2021;139:111708. doi:10.1016/j.biopha.2021.111708

25. Russo M, Della Sala A, Tocchetti CG, et al. Metabolic aspects of anthracycline cardiotoxicity. Curr Treat Options Oncol. 2021;22 (2):18. doi:10.1007/s11864-020-00812-1

26. Armenian SH, Hudson MM, Mulder RL, et al. Recommendations for cardiomyopathy surveillance for survivors of childhood cancer: a report from the International Late Effects of Childhood Cancer Guideline Harmonization Group. Lancet Oncol. 2015;16(3):e12336. doi:10.1016/S1470-2045(14)70409-7

27. Garcia-Pavia P, Kim Y, Restrepo-Cordoba MA, et al. Genetic variants associated with cancer therapy-induced cardiomyopathy. Circulation. 2019;140(1):31-41. doi:10.1161/CIRCULATIONAHA.118.037934

28. Hoff DD, Layard MW, Basa P, et al. Risk factors for doxorubicin-induced congestive heart failure. Ann Intern Med. 1979;91(5):710-717. doi:10.7326/0003-4819-91-5-710

29. Bielack SS, Erttmann R, Winkler K, et al. Doxorubicin: effect of different schedules on toxicity and anti-tumor efficacy. Eur J Cancer Clin Oncol. 1989;25(5):873-882. doi:10.1016/0277-5379(89)90135-1

30. Lipshultz SE, Miller TL, Lipsitz SR, et al. Continuous versus bolus infusion of doxorubicin in children with ALL: long-term cardiac outcomes. Pediatrics. 2012;130(6):1003-1011. doi:10.1542/ peds.2012-0727

31. Cowgill JA, Francis SA, Sawyer DB. Anthracycline and peripartum cardiomyopathies. Circ Res. 2019;124(11):1633-1646. doi:10.1161/ CIRCRESAHA.119.313577

32. Menna P, Salvatorelli E. Primary prevention strategies for anthracycline cardiotoxicity: a brief overview. Chemotherapy. 2017;62 (3):159-168. doi:10.1159/000455823

33. Bansal N, Adams MJ, Ganatra S, et al. Strategies to prevent anthracycline-induced cardiotoxicity in cancer survivors. Cardiooncology. 2019;5:18. doi:10.1186/s40959-019-0054-5

34. Kopp LM, Womer RB, Schwartz CL, et al. Effects of dexrazoxane on doxorubicin-related cardiotoxicity and second malignant neoplasms in children with osteosarcoma: a report from the children's oncology group. Cardiooncology. 2019;5:15. doi:10.1186/s40959-019-0050-9

35. Cheuk DK, Sieswerda E, van Dalen EC, et al. Medical interventions for treating anthracycline-induced symptomatic and asymptomatic cardiotoxicity during and after treatment for childhood cancer. Cochrane Database Syst Rev. 2016;(8):CD008011. doi:10.1002/ 14651858.CD008011.pub3
36. Bock MJ, Pahl E, Rusconi PG, et al. Cancer recurrence and mortality after pediatric heart transplantation for anthracycline cardiomyopathy: a report from the Pediatric Heart Transplant Study (PHTS) group. Pediatr Transplant. 2017;21(5):e12923. doi:10.1111/ petr. 12923

37. Shugh SB, Ryan TD. Heart transplantation in survivors of childhood cancer. Transl Pediatr. 2019;8(4):314-321. doi:10.21037/ tp.2019.06.02

38. Curigliano G, Lenihan D, Fradley M, et al. Management of cardiac disease in cancer patients throughout oncological treatment: ESMO consensus recommendations. Ann Oncol. 2020;31(2):171-190. doi:10.1016/j.annonc.2019.10.023

39. Thornley P, Vicente M, MacDonald A, et al. Causes and frequencies of reoperations after endoprosthetic reconstructions for extremity tumor surgery: a systematic review. Clin Orthop Relat Res. 2019;477(4):894-902. doi:10.1097/CORR.0000000000000630

40. Grinberg SZ, Posta A, Weber KL, et al. Limb salvage and reconstruction options in osteosarcoma. Adv Exp Med Biol. 2020;1257:13-29. doi:10.1007/978-3-030-43032-0 2

41. Clemens E, van den Heuvel-eibrink MM, Mulder RL, et al. Recommendations for ototoxicity surveillance for childhood, adolescent, and young adult cancer survivors: a report from the International Late Effects of Childhood Cancer Guideline Harmonization Group in collaboration with the PanCare Consortium. Lancet Oncol. 2019;20(1):e29-e41. doi:10.1016/ S1470-2045(18)30858-1

42. Romano A, Capozza MA, Mastrangelo S, et al. Assessment and management of platinum-related ototoxicity in children treated for cancer. Cancers. 2020;12(5):1266. doi:10.3390/cancers 12051266

43. van As JW, van den Berg H, van Dalen EC. Platinum-induced hearing loss after treatment for childhood cancer. Cochrane Database Syst Rev. 2016;2016(8):CD010181. doi:10.1002/ 14651858.CD010181.pub2

44. Moke DJ, Luo C, Millstein J, et al. Prevalence and risk factors for cisplatin-induced hearing loss in children, adolescents, and young adults: a multi-institutional North American cohort study. Lancet Child Adolesc Health. 2021;5(4):274-283. doi:10.1016/S23524642(21)00020-1

45. Freyer DR, Brock PR, Chang KW, et al. Prevention of cisplatin-induced ototoxicity in children and adolescents with cancer: a clinical practice guideline. Lancet Child Adolesc Health. 2020;4 (2):141-150. doi:10.1016/S2352-4642(19)30336-0

46. Skinner R. Late renal toxicity of treatment for childhood malignancy: risk factors, long-term outcomes, and surveillance. Pediatr Nephrol. 2018;33(2):215-225. doi:10.1007/s00467-017-3662-z

47. Howard SC, McCormick J, Pui CH, et al. Preventing and managing toxicities of high-dose methotrexate. Oncologist. 2016;21 (12):1471-1482. doi:10.1634/theoncologist.2015-0164

48. Foreman JW. Fanconi syndrome. Pediatr Clin Noth Am. 2019;66 (1):159-167. doi:10.1016/j.pcl.2018.09.002

49. Rossi R, Danzebrink S, Hillebrand D, et al. Ifosfamide-induced subclinical nephrotoxicity and its potentiation by cisplatinum. Med Pediatr Oncol. 1994;22(1):27-32. doi:10.1002/mpo.2950220106

50. Hanly L, Chen N, Rieder M, et al. Ifosfamide nephrotoxicity in children: a mechanistic base for pharmacological prevention. Expert Opin Drug Saf. 2009;8(2):155-168. doi:10.1517/14740330 902808169

51. Krull KR, Hardy KK, Kahalley LS, et al. Neurocognitive outcomes and interventions in long-term survivors of childhood cancer. J Clin Oncol. 2018;36(21):2181-2189. doi:10.1200/JCO.2017.76.4696

52. Avan A, Postma TJ, Ceresa C, et al. Platinum-induced neurotoxicity and preventive strategies: past, present, and future. Oncologist. 2015;20(4):411-432. doi:10.1634/theoncologist.2014-0044

53. Epstein JB, Thariat J, Bensadoun RJ, et al. Oral complications of cancer and cancer therapy: from cancer treatment to survivorship. $C A$ Cancer J Clin. 2012;62(6):400-422. doi:10.3322/caac.21157 
54. Laws HJ, Baumann U, Bogdan C, et al. Vaccinations in immunodeficiency. Application notes for vaccinations recommended by the Ständige Impfkommission. (III) Vaccinations in hematological and oncological diseases (antineoplastic therapy, stem cell transplantation), organ transplantation, and asplenia. Bundesgesundheitsblatt Gesundheitsforschung Gesundheitsschutz. 2020;63(5):588-644. German. doi:10.1007/s00103-020-03123-w

55. Rubin LG, Levin MJ, Ljungman P, et al. IDSA clinical practice guideline for vaccination of the immunocompromised host. Clin Infect Dis. 2014;58(3):309-318. doi:10.1093/cid/cit816

56. Cesaro S, Giacchino M, Fioredda F, et al. Guidelines on vaccinations in paediatric haematology and oncology patients. Biomed Res Int. 2014;2014:707691. doi:10.1155/2014/707691

57. Pittet LF, Posfay-Barbe KM. Vaccination of immune compromised children-an overview for physicians. Eur J Pediatr. 2021;180 (7):2035-2047. doi:10.1007/s00431-021-03997-1

58. Bader MS. Herpes zoster: diagnostic, therapeutic, and preventive approaches. Postgrad Med. 2013;125(5):78-91. doi:10.3810/ pgm.2013.09.2703

59. Oktay K, Harvey BE, Partridge AH, et al. Fertility preservation in patients with cancer: ASCO clinical practice guideline update. J Clin Oncol. 2018;36(19):1994-2001. doi:10.1200/JCO.2018.78.1914

60. Byrne J, Fears TR, Gail MH, et al. Early menopause in long-term survivors of cancer during adolescence. Am J Obstet Gynecol. 1992;166(3):788-793. doi:10.1016/0002-9378(92)91335-8

61. Fauske L, Bondevik H, Bruland ØS, et al. Negative and positive consequences of cancer treatment experienced by long-term osteosarcoma survivors: a qualitative study. Anticancer Res. 2015;35 (11):6081-6090.

62. Edelmann MN, Daryani VM, Bishop MW, et al. Neurocognitive and patient-reported outcomes in adult survivors of childhood osteosarcoma. JAMA Oncol. 2016;2(2):201-208. doi:10.1001/ jamaoncol.2015.4398

63. Holzer LA, Huyer N, Friesenbichler J, et al. Body image, self-esteem, and quality of life in patients with primary malignant bone tumors. Arch Orthop Trauma Surg. 2020;140(1):1-10. doi:10.1007/s00402019-03205-8
64. Weschenfelder W, Gast-Froehlich S, Spiegel C, et al. Factors influencing quality of life, function, reintegration and participation after musculoskeletal tumour operations. BMC Cancer. 2020;20(1):351. doi:10.1186/s12885-020-06837-x

65. van Waas M, Neggers SJ, van der Lelij AJ, et al. The metabolic syndrome in adult survivors of childhood cancer, a review. J Pediatr Hematol Oncol. 2010;32(3):171-179. doi:10.1097/MPH.0b013 e3181d419c3

66. Chueh HW, Yoo JH. Metabolic syndrome induced by anticancer treatment in childhood cancer survivors. Ann Pediatr Endocrinol Metab. 2017;22(2):82-89. doi:10.6065/apem.2017.22.2.82

67. Calaminus G, Jenney M, Hjorth L, et al. Quality of life of patients with osteosarcoma in the European American Osteosarcoma Study-1 (EURAMOS-1): development and implementation of a questionnaire substudy. JMIR Res Protoc. 2019;8(9):e14406. doi:10.2196/14406

68. Omer N, Le Deley MC, Piperno-Neumann S, et al. Phase-II trials in osteosarcoma recurrences: a systematic review of past experience. Eur J Cancer. 2017;75:98-108. doi:10.1016/j.ejca.2017.01.00

69. Gill J, Gorlick R. Advancing therapy for osteosarcoma. Nat Rev Clin Oncol. 2021;18(10):609-624. doi:10.1038/s41571-021-00519-8

70. Meyers PA. Muramyl tripeptide-phosphatidyl ethanolamine encapsulated in liposomes (L-MTP-PE) in the treatment of osteosarcoma. Adv Exp Med Biol. 2020;257:133-139. doi:10.1007/978-3-03043032-0 11

71. Mifamurtide: osteosarcoma: ineffective and harmful. Prescrire Int. 2011;20(115):89.

72. Chow EJ, Antal Z, Constine LS, et al. New agents, emerging late effects, and the development of precision survivorship. J Clin Oncol. 2018;36(21):2231-2240. doi:10.1200/JCO.2017.76.4647

73. Williams AM, Liu Q, Bhakta N, et al. Rethinking success in pediatric oncology: beyond 5-year survival. J Clin Oncol. 2021;39 (20):2227-2231. doi:10.1200/JCO.20.03681

74. Langer T, Stöhr W, Bielack S, et al. Late effects surveillance system for sarcoma patients. Pediatr. Blood Cancer. 2004, 42(4):373-9. doi:10.1002/pbc. 10325

\section{Publish your work in this journal}

Cancer Management and Research is an international, peer-reviewed open access journal focusing on cancer research and the optimal use of preventative and integrated treatment interventions to achieve improved outcomes, enhanced survival and quality of life for the cancer patient.
The manuscript management system is completely online and includes a very quick and fair peer-review system, which is all easy to use. Visit http://www.dovepress.com/testimonials.php to read real quotes from published authors. 